\title{
GEOMETRIC CALIBRATION OF THERMOGRAPHIC CAMERAS
}

\author{
Thomas Luhmann* Julia Ohm, Johannes Piechel, Thorsten Roelfs \\ Institute for Applied Photogrammetry and Geoinformatics, Jade University of Applied Sciences Oldenburg, Ofener Str.
} 16-19, D-26121 Oldenburg, Germany (thomas.luhmann@jade-hs.de)

\section{Commission V, WG V/5}

KEY WORDS: Thermography, Thermal Imaging, Calibration, Accuracy, Application

\begin{abstract}
:
The paper gives an overview of thermal imaging sensors for photogrammetric close-range applications. In particular, it presents results of the geometric calibration of thermographic cameras as they are used for building inspection and material testing. Two different test fields have been designed providing point targets that are visible in the thermal spectral band of the cameras.

Five different cameras have been investigated. Four of them have solid state sensors with pixel sizes between 25 and $40 \mu \mathrm{m}$. One camera is working in scanning mode. The lenses for thermographic cameras are made of Germanium. Conventional imaging configurations (typically 20 images) have been used for camera calibration. Standard parameters for principal distance, principal point, radial distortion, decentring distortion, affinity and shear have been introduced into the self-calibrating bundle adjustment. All measured points are introduced as weighted control points. Image coordinates have been measured either in the professional software package AICON 3D Studio (ellipse operators), or in the IAPG software system Stereomess (least-squares template matching).
\end{abstract}

The calibration results differ significantly from camera to camera. All lenses show relatively large decentring distortion and deviations from orthogonality of the image coordinate axes. Using the plane test field with lamps, the average image precision is $3 / 10^{\text {th }}$ of a pixel while the $3 \mathrm{D}$ test field with circular reflecting targets results in imaging errors of $1 / 20^{\text {th }}$ pixel.

\section{CAMERA TECHNOLOGY}

\subsection{Physical background}

Thermographic cameras are widely used in the fields of material testing, quality control and building monitoring. In all of these cases the radiometric information about temperature distribution is of major interest. Geometric applications are still rare, hence camera developers and suppliers still show little interest in photogrammetric techniques. However, with increasing resolution of thermographic cameras the geometric processing of the image data will become more important.

The specific spectral emission $M_{\lambda}$ of an object is defined by Planck's emission law as a function of absolute temperature and wavelength (Wolfe \& Zissis, 1985):

$$
M_{\lambda}\left[\frac{W}{c m^{2} \cdot \mu m}\right]=\frac{c_{1}}{\lambda^{5}} \frac{1}{\exp \left(\frac{c_{2}}{\lambda \cdot T}\right)-1}
$$

where

$$
\begin{array}{ll}
c_{1}: & \text { 1. emission constant }=3,7418 \cdot 10^{4} \mathrm{~W} \cdot \mathrm{cm}^{-2} \cdot \mu \mathrm{m}^{4} \\
c_{2}: & 2 . \text { emission constant }=1,4388 \cdot 10^{4} \mathrm{~K} \cdot \mu \mathrm{m} \\
T: & \text { absolute temperature }[\mathrm{K}] \\
\lambda: & \text { wave length }[\mu \mathrm{m}]
\end{array}
$$

Fig. 1 shows the well known diagram of specific spectral emission for different absolute temperatures according to equation (1). It makes clear that only objects with a temperature of more than about $1000 \mathrm{~K}$ are emitting electro-magnetic radiation visible for the human eye or conventional cameras.
The maximum of the specific emission is described by Wien's displacement law:

$\lambda_{\max }=\frac{2897.8}{T}$

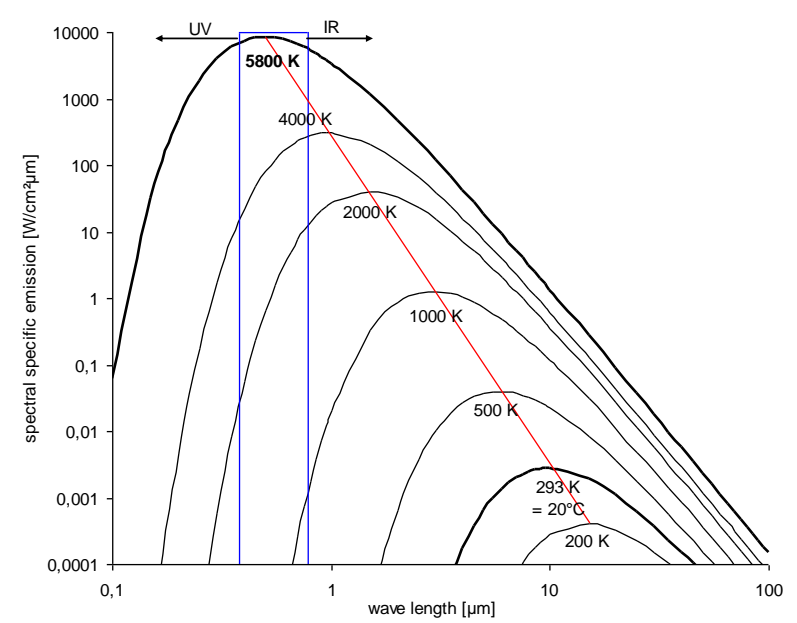

Fig. 1: Spectral specific emission

In equation (2) the value 2897.8 is denoted as the Wien displacement constant. The formula states that the wavelength of maximum emission of an object is a reciprocal function of the temperature, hence higher temperatures yield lower wave lengths (Dereniak \& Boreman, 1996). As an example, the sun has an average temperature of about $5800 \mathrm{~K}$, the resulting wavelength is $0.5 \mu \mathrm{m}$, thus in the yellow band of the visible 
light spectrum. As a second example, an object of $20^{\circ} \mathrm{C}$ (=293 K), the resulting wavelength is about $10 \mu \mathrm{m}$.

Consequently thermographic cameras need imaging sensors that are sensitive to wavelengths usually between 2.5 and $15 \mu \mathrm{m}$. Depending on the detector technology (see section 1.2) temperatures between -30 and $+400^{\circ} \mathrm{C}$ can be detected by common thermographic cameras.

The geometric resolution of imaging devices is limited by diffraction. The diameter of the Airy disk $d$ depends on the aperture (f-number $k$ ) and the wavelength:

$d=2.44 \cdot \lambda k$

As an example, geometric resolution at a wavelength of $\lambda=10 \mu \mathrm{m}$ at $\mathrm{f}$-number $k=2$ is limited to about $48 \mu \mathrm{m}$. Equation (3) makes clear that the pixel sizes of thermal sensors can be much larger than for standard RGB cameras. In fact most thermal imaging sensors provide pixel sizes between 30 and $50 \mu \mathrm{m}$.

\subsection{Detectors}

Sensors for thermal cameras are either quantum detectors or thermal detectors (Nolting, 2007). Quantum detectors are based on the inner photo-electric effect where electrons are set free between two layers of a semi-conductor device. Quantum detectors are very sensitive $( \pm 0.01 \mathrm{~K})$ and fast, but need an external cooling system (Peltier or Sterling elements) (Fouad \& Richter, 2008).

Thermal detectors use the effect that a temperature change of the detector element leads to a change of the electrical properties of the detector, e.g. resistance or charges (Bauer, 2008). These changes can be measured and transformed into intensity values. Thermal detectors are available in different designs such as pyro-electric detectors or bolometers. Thermal detectors are less sensitive $( \pm 0.1 \mathrm{~K})$ and slower than quantum detectors, but do not need any cooling elements. Hence they are less expensive und usually applied for applications like building monitoring.

As state-of-the-art typical thermal array sensors are available with $320 \times 240$ pixels up to $640 \times 320$ pixels. Sensor sizes then yield about up to $20-30 \mathrm{~mm}$ in each direction. Newest camera developments show pixel numbers of up to $1280 \times 960$

In principle thermographic cameras built on solid state sensors can be handled as standard photogrammetric cameras. Due to the longer wavelengths the lenses of thermographic cameras are made of Germanium which makes them extremely expensive. These lenses are optimized for radiometric resolution, thus geometric precision or minimal distortion are of less interest.

\section{TEST FIELDS FOR CALIBRATION}

\subsection{Plane testfield with lamps}

A plane testfield provided by the University of Dessau consists of 57 small lamps that warm up when switched on. The dimension of the wooden plate is about $1000 \mathrm{~mm} \times 1000 \mathrm{~mm}$
(Fig. 2). The positions of the lamps have been measured by a theodolite system with an accuracy of about $0.2 \mathrm{~mm}$.

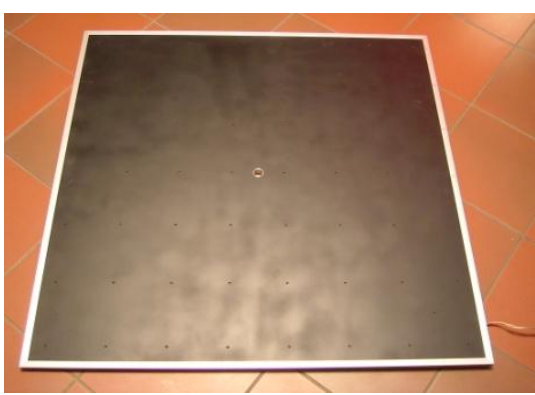

Fig. 2: Plane testfield with active lamps

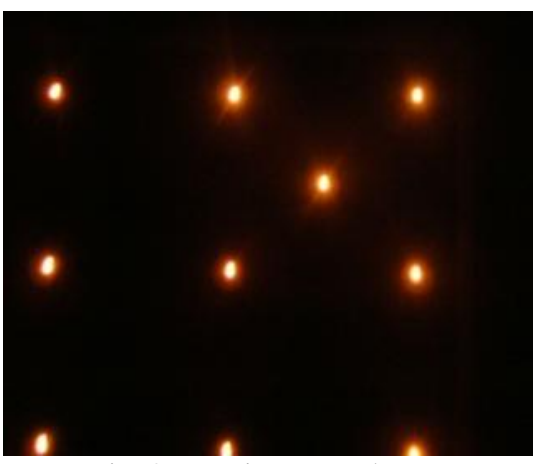

Fig. 3: Burning target lamps

The quality of the active targets is quite poor (Fig. 3). It is obvious that the centre of the targets lamps can not be measured with a precision as usually provided by photogrammetric targets. In addition, camera calibration with plane testfields is less accurate and less significant compared to 3D testfields.

\subsection{Spatial testfield with coded targets}

Since the plane testfield is not ideal for camera calibration a new design was investigated. The basic idea was to create targets that generate sufficient image contrast in the thermal spectrum. In addition, the new testfield should be mobile, easy to calibrate and cost effective without any need for artificial heating of targets.

Several experiments have been carried out in order to find a suitable combination of target material and lighting. Finally, the effect of cold sky temperature has been used to create a testfield design that fulfils the above mentioned specifications.

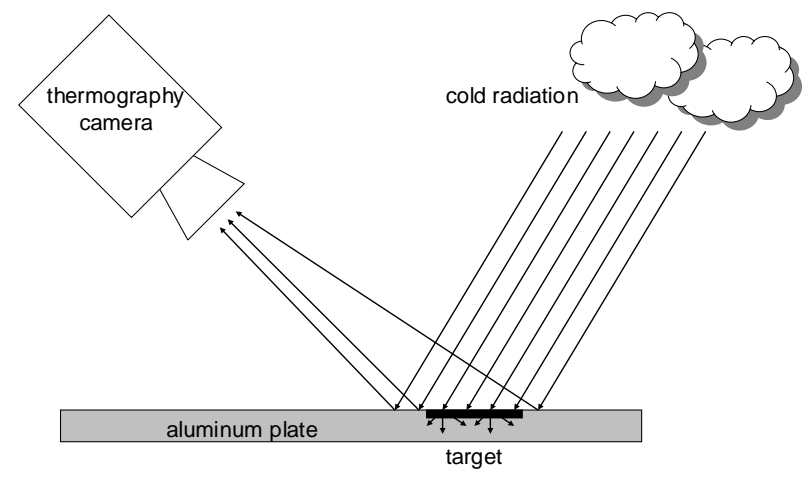

Fig. 4: Target absorption of cold sky radiation 
Fig. 4 shows the reflectance principle. Assuming diffuse reflectance of the metal testfield plate the cold temperature of space reflects on the metal surface. Since the target is made of self-adhesive foil it only emits radiation relating to its own temperature. With this principle the acquired image displays a strong contrast because targets appear bright while the surrounding areas appear dark (Fig. 5).
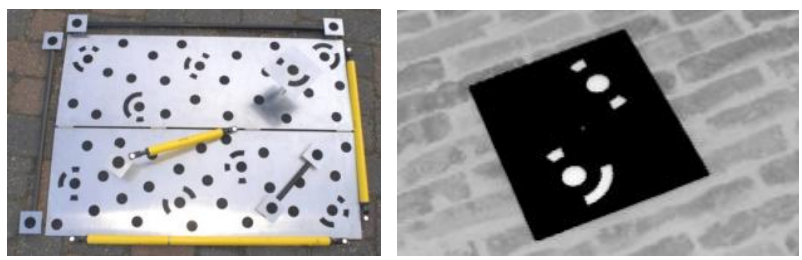

Fig. 5: Testfield and thermal image of targets

Based on the new design of target points a mobile testfield has been built. It consists of about 17 coded targets and 35 uncoded targets, and additional height points. Additional scale bars with the same type of targets can be mounted onto the testfield. Due to the target design a conventional photogrammetric calibration of the testfield is possible which yields control points coordinates.

The size of the testfield is about $1000 \mathrm{~mm} \times 700 \mathrm{~mm} \times 200 \mathrm{~mm}$. The accuracy of control points after measurement with a highresolution digital camera and bundle adjustment is estimated to $8 \mu \mathrm{m}$ in object space.

\section{CALIBRATION RESULTS}

\subsection{Cameras}

Table 1 summarizes the technical data of four thermographic cameras that are included in the test. A fifth camera works with a rotation mirror device for image scanning. Due to the instable mechanical rotation and the non-perspective imaging model this camera has been eliminated from further tests for the time being.

The investigated cameras (see Fig. 6) show more or less similar technical data. However, due to the different detector elements their perfomance differs as well as their market prices.

\begin{tabular}{|l|l|l|l|l|}
\hline & $\begin{array}{l}\text { FLIR } \\
\text { InfraCam }\end{array}$ & $\begin{array}{l}\text { Testo } \\
880-3\end{array}$ & $\begin{array}{l}\text { FLIR } \\
\text { B200 }\end{array}$ & $\begin{array}{l}\text { InfraTec } \\
\text { VarioCam }\end{array}$ \\
\hline pixels & $240 \times 240$ & $320 \times 240$ & $320 \times 240$ & $384 \times 288$ \\
\hline pixel size & $0.025 \mathrm{~mm}$ & $0.035 \mathrm{~mm}$ & $0.04 \mathrm{~mm}$ & $0.035 \mathrm{~mm}$ \\
\hline focal length & $10 \mathrm{~mm}$ & $10 \mathrm{~mm}$ & $18 \mathrm{~mm}$ & $11 \mathrm{~mm}$ \\
\hline $\begin{array}{l}\text { thermal } \\
\text { resolution }\end{array}$ & $\pm 0.2^{\circ} \mathrm{C}$ & $<0.3^{\circ} \mathrm{C}$ & $\pm 0.08^{\circ} \mathrm{C}$ & $\begin{array}{l}0.08- \\
0.05^{\circ} \mathrm{C}\end{array}$ \\
\hline price $[€]$ & ca. 4000 & ca. 6500 & ca. 9000 & ca. 19000 \\
\hline
\end{tabular}

Table 1: Investigated thermographic cameras

\subsection{Calibration results}

Each camera was calibrated according to standard imaging configurations (Luhmann et al., 2006) with about 20 multiconvergent images. Both testfields as explained in section 2 have been used (Fig. 7). The plane testfield with burning lamps can be measured inside a lab while the reflective 3D testfield has to be used in open air.
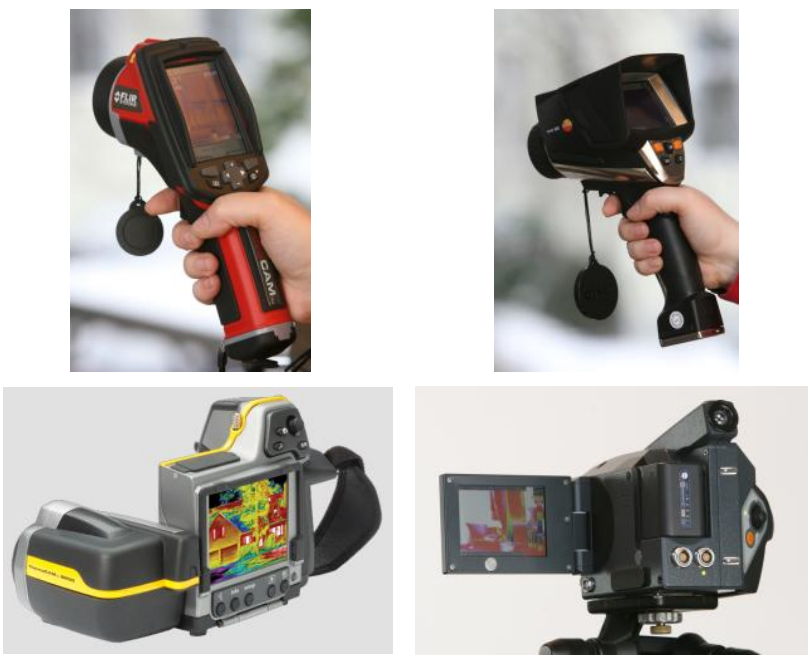

Fig. 6: Investigated thermographic cameras
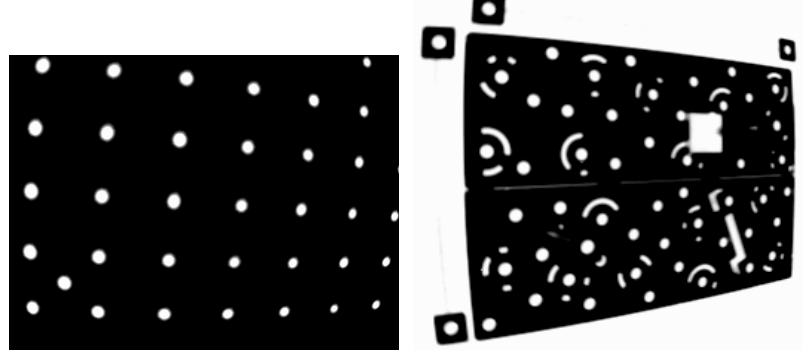

Fig. 7: Thermal images acquired from plane testfield (left) and spatial testfield (right)

Image measurement and bundle adjustment were performed with AICON 3D Studio. The calibration results, derived from the spatial testfield, are listed in Tables 2-5. The plane testfield leads to similar results. However the precision of the estimated parameters is reduced and therefore they are not listed here.

\begin{tabular}{|l|r|r|}
\hline parameter & \multicolumn{1}{|c|}{ value } & \multicolumn{1}{|c|}{ standard dev. } \\
\hline principal distance $\mathrm{c}$ & $-13.8971 \mathrm{~mm}$ & 0.0104 \\
\hline principal point $\mathrm{x}_{0}^{\prime}$ & $-0.2940 \mathrm{~mm}$ & 0.0188 \\
\hline principal point $\mathrm{y}_{0}^{\prime}$ & $-0.3430 \mathrm{~mm}$ & 0.0197 \\
\hline radial-sym. distortion $\mathrm{A}_{1}$ & $-2.80 \cdot 10^{-3}$ & $1.33 \cdot 10^{-4}$ \\
\hline radial-sym. distortion $\mathrm{A}_{2}$ & $6.86 \cdot 10^{-7}$ & $2.03 \cdot 10^{-5}$ \\
\hline radial-sym. distortion $\mathrm{A}_{3}$ & $7.76 \cdot 10^{-7}$ & $9.52 \cdot 10^{-7}$ \\
\hline decentring distortion $\mathrm{B}_{1}$ & $1.91 \cdot 10^{-5}$ & $2.10 \cdot 10^{-5}$ \\
\hline decentring distortion $\mathrm{B}_{2}$ & $-2.69 \cdot 10^{-5}$ & $2.25 \cdot 10^{-5}$ \\
\hline affinity $\mathrm{C}_{1}$ & $6.84 \cdot 10^{-4}$ & $1.99 \cdot 10^{-4}$ \\
\hline shear $\mathrm{C}_{2}$ & $-2.22 \cdot 10^{-4}$ & $2.21 \cdot 10^{-4}$ \\
\hline
\end{tabular}

Table 2: Calibrated camera parameters (FLIR InfraCam) 


\begin{tabular}{|l|r|r|}
\hline parameter & \multicolumn{1}{|c|}{ value } & \multicolumn{1}{l|}{ standard dev. } \\
\hline principal distance $\mathrm{c}$ & $-19.9373 \mathrm{~mm}$ & 0.0297 \\
\hline principal point $\mathrm{x}_{0}^{\prime}$ & $-0.1571 \mathrm{~mm}$ & 0.0524 \\
\hline principal point $\mathrm{y}_{0}^{\prime}$ & $0.2110 \mathrm{~mm}$ & 0.0376 \\
\hline radial-sym. distortion $\mathrm{A}_{1}$ & $-7.25 \cdot 10^{-4}$ & $1.20 \cdot 10^{-4}$ \\
\hline radial-sym. distortion $\mathrm{A}_{2}$ & $-9.59 \cdot 10^{-6}$ & $7.19 \cdot 10^{-6}$ \\
\hline radial-sym. distortion $\mathrm{A}_{3}$ & $1.86 \cdot 10^{-7}$ & $1.28 \cdot 10^{-7}$ \\
\hline decentring distortion $\mathrm{B}_{1}$ & $5.12 \cdot 10^{-5}$ & $1.22 \cdot 10^{-5}$ \\
\hline decentring distortion $\mathrm{B}_{2}$ & $-3.51 \cdot 10^{-5}$ & $1.72 \cdot 10^{-5}$ \\
\hline affinity $\mathrm{C}_{1}$ & $7.50 \cdot 10^{-4}$ & $3.16 \cdot 10^{-4}$ \\
\hline shear $\mathrm{C}_{2}$ & $8.18 \cdot 10^{-4}$ & $2.96 \cdot 10^{-4}$ \\
\hline
\end{tabular}

Table 3: Calibrated camera parameters (Testo $880-3$ )

\begin{tabular}{|l|r|r|}
\hline parameter & \multicolumn{1}{|c|}{ value } & \multicolumn{1}{l|}{ standard dev. } \\
\hline principal distance $\mathrm{c}$ & $-36.9443 \mathrm{~mm}$ & 0.0223 \\
\hline principal point $\mathrm{x}_{0}^{\prime}$ & $1.4445 \mathrm{~mm}$ & 0.0574 \\
\hline principal point $\mathrm{y}_{0}^{\prime}$ & $-1.8332 \mathrm{~mm}$ & 0.0420 \\
\hline radial-sym. distortion $\mathrm{A}_{1}$ & $-2.69 \cdot 10^{-4}$ & $1.94 \cdot 10^{-5}$ \\
\hline radial-sym. distortion $\mathrm{A}_{2}$ & $-7.47 \cdot 10^{-7}$ & $6.94 \cdot 10^{-7}$ \\
\hline radial-sym. distortion $\mathrm{A}_{3}$ & $2.79 \cdot 10^{-9}$ & $7.40 \cdot 10^{-9}$ \\
\hline decentring distortion $\mathrm{B}_{1}$ & $-2.78 \cdot 10^{-4}$ & $5.21 \cdot 10^{-6}$ \\
\hline decentring distortion $\mathrm{B}_{2}$ & $-1.72 \cdot 10^{-5}$ & $6.18 \cdot 10^{-6}$ \\
\hline affinity $\mathrm{C}_{1}$ & $-6.11 \cdot 10^{-4}$ & $2.60 \cdot 10^{-4}$ \\
\hline shear $\mathrm{C}_{2}$ & $-8.66 \cdot 10^{-4}$ & $2.61 \cdot 10^{-4}$ \\
\hline
\end{tabular}

Table 4: Calibrated camera parameters (FLIR B200)

\begin{tabular}{|l|r|r|}
\hline parameter & \multicolumn{1}{|c|}{ value } & \multicolumn{1}{|c|}{ standard dev. } \\
\hline principal distance $\mathrm{c}$ & $-11.8188 \mathrm{~mm}$ & 0.0014 \\
\hline principal point $\mathrm{x}_{0}^{\prime}$ & $0.0201 \mathrm{~mm}$ & 0.0013 \\
\hline principal point $\mathrm{y}_{0}^{\prime}$ & $0.1400 \mathrm{~mm}$ & 0.0012 \\
\hline radial-sym. distortion $\mathrm{A}_{1}$ & $-2.41 \cdot 10^{-3}$ & $4.93 \cdot 10^{-6}$ \\
\hline radial-sym. distortion $\mathrm{A}_{2}$ & $8.76 \cdot 10^{-6}$ & $1.83 \cdot 10^{-7}$ \\
\hline radial-sym. distortion $\mathrm{A}_{3}$ & $-2.67 \cdot 10^{-8}$ & $2.05 \cdot 10^{-9}$ \\
\hline decentring distortion $\mathrm{B}_{1}$ & $5.23 \cdot 10^{-5}$ & $1.11 \cdot 10^{-6}$ \\
\hline decentring distortion $\mathrm{B}_{2}$ & $-1.29 \cdot 10^{-5}$ & $1.14 \cdot 10^{-6}$ \\
\hline affinity $\mathrm{C}_{1}$ & $-7.79 \cdot 10^{-5}$ & $2.47 \cdot 10^{-5}$ \\
\hline shear $\mathrm{C}_{2}$ & $1.85 \cdot 10^{-4}$ & $1.91 \cdot 10^{-5}$ \\
\hline
\end{tabular}

Table 5: Calibrated camera parameters (InfraTec VarioCam)

The cameras FLIR InfraCam, Testo 880-3 and FLIR B200 show relatively weak results in terms of image measurement precision and standard deviations of principal point and principal distance. Compared to the given focal length (as taken from data sheets) the principal distance differs significantly. In the case of the FLIR B200 the principal point shift amounts to more than $1.4 \mathrm{~mm}$, which corresponds to more than 35 pixels. All cameras show relatively large radial distortion values as depicted in Fig. 8.
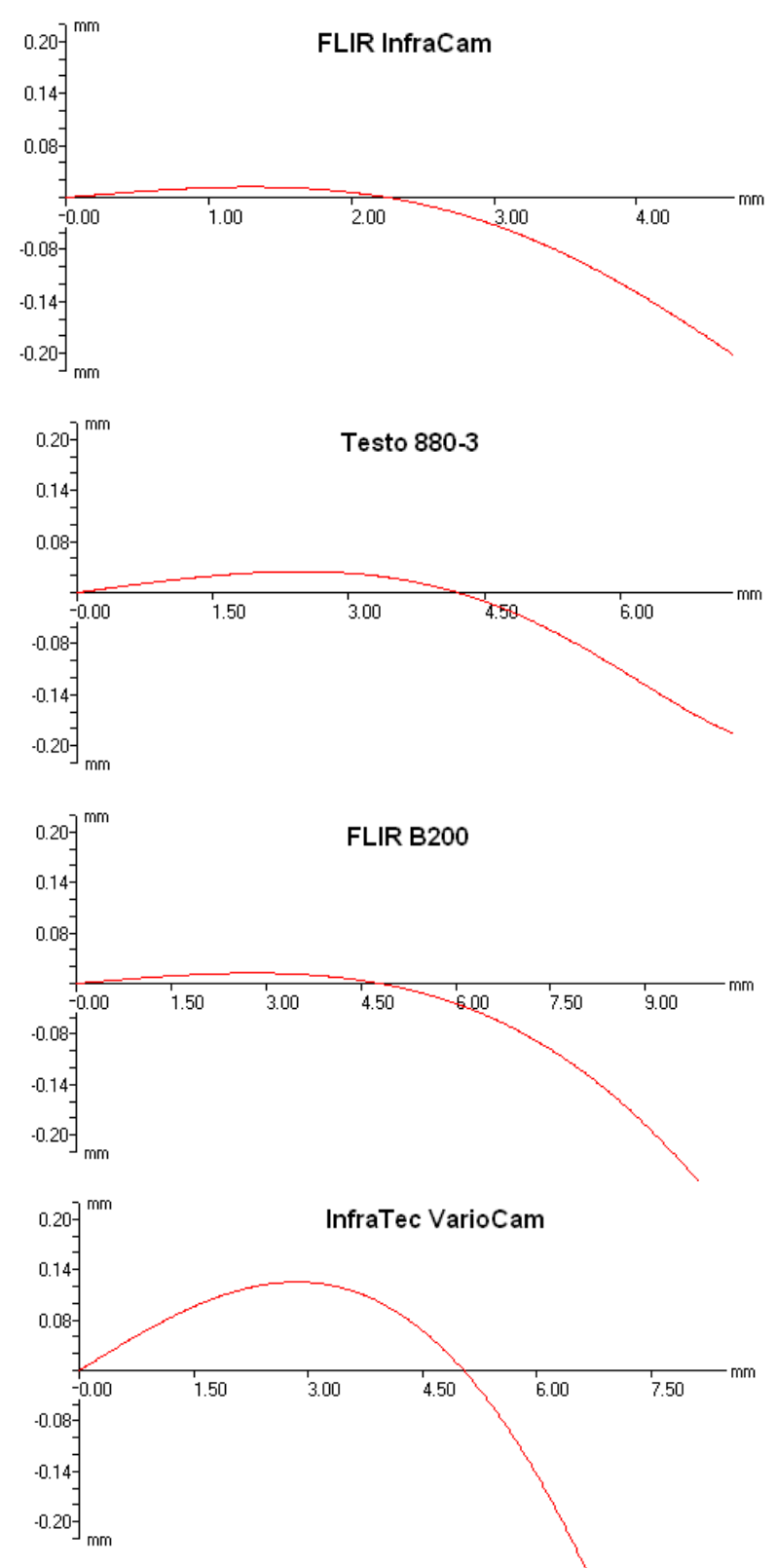

Fig. 8: Distortion curves

The camera InfraTec VarioCam yields the best results in terms of precision of the estimated parameters. The precision of image point measurement lies in the order of $1 \mu \mathrm{m}$ or $1 / 30^{\text {th }}$ of a pixel. For this camera the principal point shows moderate shift with respect to the centre of the image, and the principal distance is close to the given focal length.

The resulting precision in object space is estimated by RMS values of adjusted object coordinates. External reference values, for instance calibrated lengths, are not available in this test. Table 6 summarizes the results in object space. As expected from the calibration quality discussed above, the three cameras FLIR InfraCam, Testo 880-3 and FLIR B200 yield RMS values (1 sigma) of about 0.11 to $0.24 \mathrm{~mm}$, which corresponds to about 1:6000 of the largest object diameter. The Infratec VarioCam results in RMS values of 0.03 to $0.06 \mathrm{~mm}$, corresponding to about 1:20000. 


\begin{tabular}{|l|l|l|l|}
\hline camera & $\mathrm{X}$ & $\mathrm{Y}$ & $\mathrm{Z}$ \\
\hline FLIR InfraCam & $0.110 \mathrm{~mm}$ & $0.118 \mathrm{~mm}$ & $0.143 \mathrm{~mm}$ \\
\hline Testo $880-3$ & $0.137 \mathrm{~mm}$ & $0.160 \mathrm{~mm}$ & $0.236 \mathrm{~mm}$ \\
\hline FLIR B200 & $0.148 \mathrm{~mm}$ & $0.185 \mathrm{~mm}$ & $0.145 \mathrm{~mm}$ \\
\hline Infratec VarioCam & $0.038 \mathrm{~mm}$ & $0.029 \mathrm{~mm}$ & $0.062 \mathrm{~mm}$ \\
\hline
\end{tabular}

Table 6: RMS 1-sigma values of object coordinates

\section{APPLICATIONS}

If thermographic cameras are calibrated in terms of the geometric imaging model they can be used for a variety of practical applications (Kaplan, 2007).

\subsection{D processing}

For two-dimensional purposes thermal images can be resampled to distortion-free images. As an example Fig. 9 shows the original thermal image taken with the InfraTec VarioCam camera. Radial distortion is clearly visible. Fig. 10 shows the same image after correction of distortion. Geometrically corrected thermographic imagery can be used as thermal orthophotos, maps or mosaics, or as precise texture images for 3D city or building models.

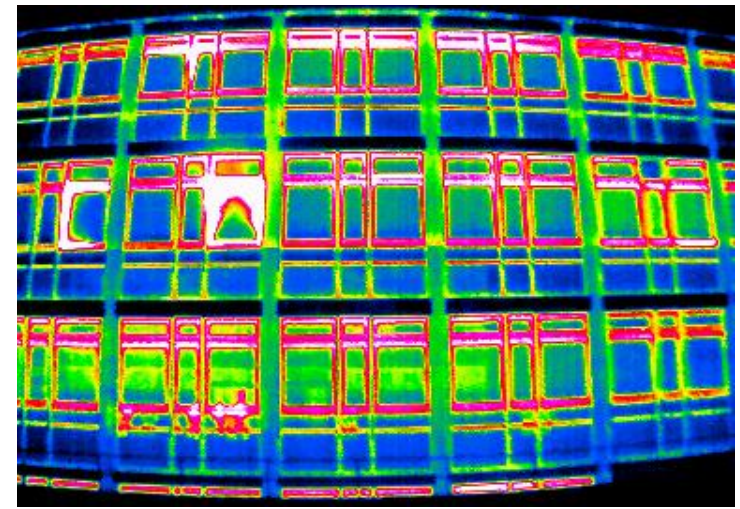

Fig. 9: Original thermal image (camera: InfraTec VarioCam)

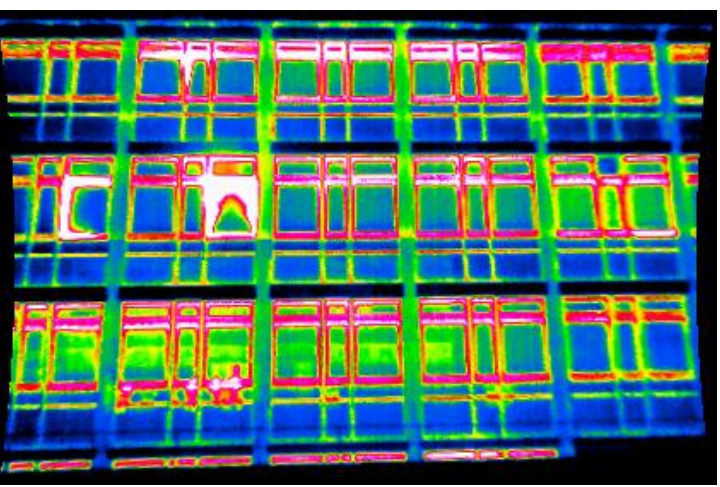

Fig. 10: Distortion-free thermal image

It has to be pointed out that modified thermographic images often can not be processed by those software packages that are provided with a specific camera system. As an example, the FLIR software package allows for post-processing of original FLIR imagery, e.g. changing temperature scales or colour tables.

\subsection{D processing}

Three-dimensional applications are also possible since thermographic images can be used in the same way as conventional photogrammetric images. However, in this case it is required to match corresponding points.
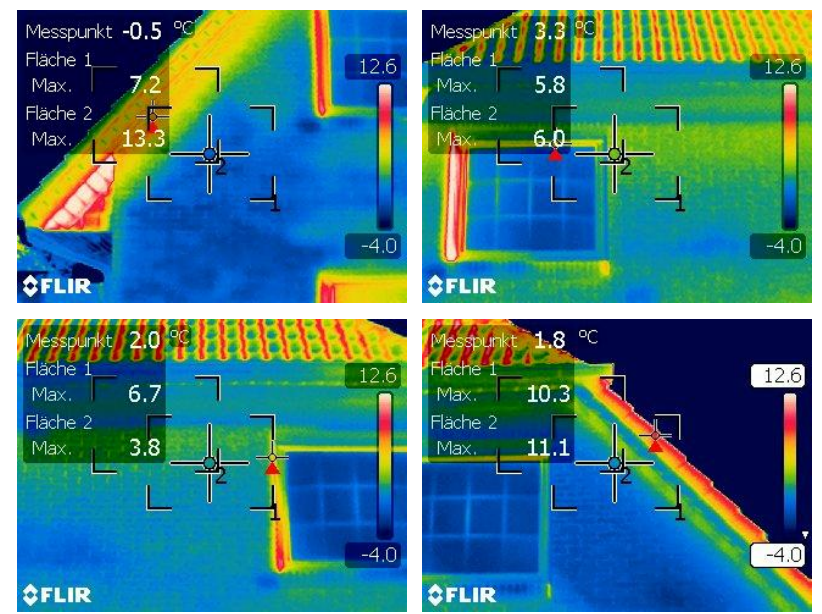

Fig. 11: Multi-image thermography

Fig. 11 shows an example of four overlapping thermographic images taken within a few minutes without changing the camera settings. It is obvious that the thermal emission of the building wall does not behave as a diffuse Lambert reflector. A number of identical object areas are displayed in different colours (temperatures).

Future investigations will concentrate on 3D modelling with thermographic imagery under consideration of radiometric object models.

\subsection{Pan sharpening}

If a high resolution panchromatic or RGB image is available in addition to a (low resolution) thermal image it is possible to apply pan-sharpening. Both image sources have to be registered (rectified) to the same geometric reference system.

Fig. 12 shows the principle image data flow for thermal pansharpening as it has been applied to the images shown below.

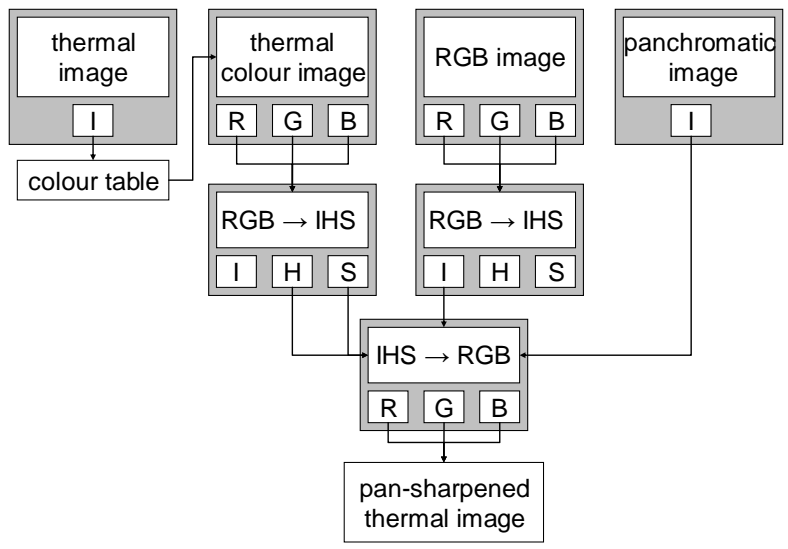

Fig. 12: Image data flow for thermal pan-sharpening 


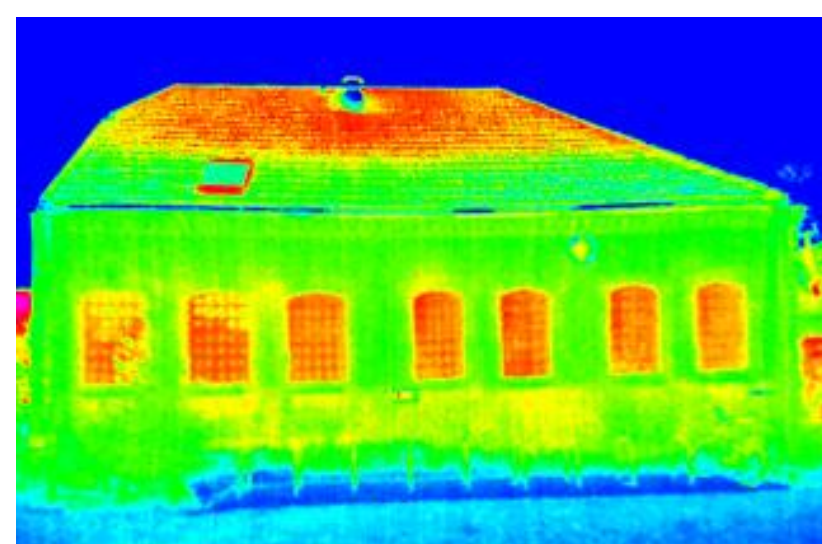

Fig. 13: Original thermal image

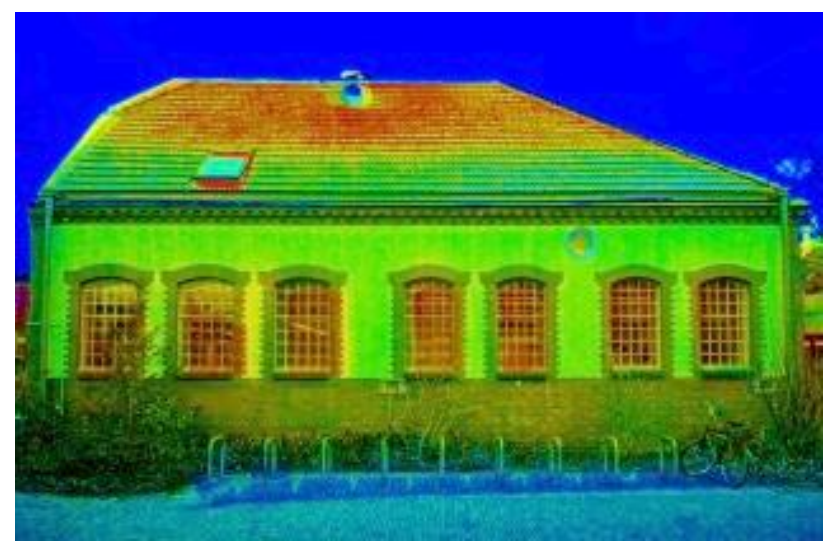

Fig. 14: Example of thermal pan-sharpening

As an example, Fig. 13 shows an original thermal image of a building façade. Fig. 14 displays the result of pan-sharpening using a high-resolution digital image taken with a Nikon D2x camera. In contrast to standard methods in remote sensing (e.g. Toet et al., 1989; Ehlers et al., 2009), applying pan-sharpening to non-planar objects in close-range photogrammetry either requires identical perspectives for each image source, or given 3D object models and full orientation parameters of each image. The example above still shows remaining geometric errors at the back parts of the roof since only a $2 \mathrm{D}$ rectification approach has been used.

It should be noted that the colours will change by this processing and an attribution of temperature levels in a following step might lead to erroneous values in small structures. However, the method of pan-sharpening is quite suitable for presentation purposes of thermographic inspections in order to illustrate details of the object.

\section{SUMMARY}

We have investigated the performance of thermographic cameras with respect to their geometric image model. Standard procedures for camera calibration can be applied to close-range thermal imagery if the cameras consist of array imaging sensors. In addition, the observed object must provide target points that are visible in the thermal spectrum. For this purpose a new testfield has been designed that uses the cold temperature from sky in order to create sufficient image contrast.

The results of camera calibration show that standard thermographic cameras yield high distortion values, and large shifts of principal point. Only one camera (Infratec VarioCam) provides an accuracy level that is comparable to RGB cameras.

Some example applications are discussed ranging from 2D image modification purposes (rectification, image mosaics) and pan-sharpening approaches up to 3D modeling tasks that uses thermographic imagery in the same way as multi-image photogrammetry. However, the radiometric models of thermal object emission have to be investigated in more detail for a better understanding of the imaging process in convergent cases.

\section{REFERENCES}

Bauer, N., (Ed.), 2008. Handbuch zur Industriellen Bildverarbeitung. Fraunhofer IRB Verlag, Stuttgart.

Dereniak, E.L., Boreman, G.D., 1996: Infrared Detectors and Systems. Wiley-Interscience, $561 \mathrm{p}$.

Ehlers, M., Klonusa, S., Åstrand, P.J., Rosso, P., 2009. Multisensor image fusion for pansharpening in remote sensing. International Journal of Image and Data Fusion, Vol. 1, No. 1, March 2010, pp. 25-45.

Fouad, N.A., Richter, T., 2008. Leitfaden Thermografie im Bauwesen. Fraunhofer IRB Verlag, Stuttgart, 127 p.

Kaplan, H., 2007. Practical Applications of Infrared Thermal Sensing and Image Equipment. SPIE Publications, 192 p.

Luhmann, T., Robson, S., Kyle, S., Harley, I., 2006: Close Range Photogrammetry. Whittles Publishing, 500 p.

Nolting, J., 2007. Detektoren für optische Strahlung. DOZ Optometrie, 4-2007, pp. 50-56.

Toet, A., van Ruyven, J.J., Valeton, J.M., 1989: Merging thermal and visual images by a contrast pyramid. Optical Engineering, 28(7), pp. 789-792.

Wolfe, W.L., Zissis, G.J., 1985: The Infrared Handbook. Environmental Research Institute of Michigan, 1700 p. 